\title{
Chemotherapy-induced gut toxicity and pain: involvement of TLRs
}

\author{
Rachel J. Gibson ${ }^{1}$ • Janet K. Coller ${ }^{2}$ • Hannah R. Wardill ${ }^{1}$ • Mark R. Hutchinson ${ }^{3}$ • \\ Scott Smid ${ }^{2}$. Joanne M. Bowen ${ }^{3}$
}

Received: 14 June 2015 / Accepted: 8 November 2015 /Published online: 19 November 2015

(C) Springer-Verlag Berlin Heidelberg 2015

\begin{abstract}
Purpose Chemotherapy-induced gut toxicity is associated with significant pain, and pain influences gut function. Tolllike receptors (TLRs) that regulate gut homeostasis are activated by tissue damage and microbes, and their altered expression following chemotherapy may change cellular responses. This study examined the interaction between chemotherapyinduced gut toxicity and pain and related these to gut TLR and glial fibrillary acidic protein (GFAP) expression.

Methods Female tumor bearing Dark Agouti rats received irinotecan $(175 \mathrm{mg} / \mathrm{kg}, n=34)$ or vehicle $(n=5)$ and were assessed over $120 \mathrm{~h}$ for gut toxicity (diarrhea, weight loss), pain (facial), and GFAP, TLR2, 4, 5, and 9 gut expression.

Results Irinotecan caused diarrhea ( $72 \%$ of animals grade $\geq$ $1)$, weight loss $(11.1 \pm 6.6 \%, P<0.0001)$, and pain $(5(0-5)$, $P<0.0001)$ all peaking at $72 \mathrm{~h}$. Higher pain scores were observed in rats with diarrhea versus those without: median (range) of $2.0(0-5)$ versus $0(0-5), P=0.01$. Irinotecan also caused a decrease in TLR4 and 5, and an increase in GFAP expression in jejuna crypt at 96 and $120 \mathrm{~h}($ all $P<0.05)$; with lower TLR4 expression associated with lower pain $(P=$ 0.012 ).
\end{abstract}

Rachel J. Gibson

rachel.gibson@adelaide.edu.au

1 Discipline of Anatomy and Pathology, School of Medicine, University of Adelaide, North Terrace, Adelaide 5005, South Australia, Australia

2 Discipline of Pharmacology, School of Medicine, University of Adelaide, Adelaide, Australia

3 Discipline of Physiology, School of Medicine, University of Adelaide, Adelaide, Australia
Conclusions The association between gut toxicity and pain suggests these toxicities are linked, possibly via TLRmediated inflammatory pathways. Further, as TLR4 and 5 expression was absent during recovery in the jejuna and GFAP expression was increased in the jejuna, this implies expression of these may be critical in the healing phase following chemotherapy. Detailed studies of gut TLRs and GFAP are now warranted.

Keywords Chemotherapy · Gut toxicity · Pain · TLR · GFAP

\section{Introduction}

Chemotherapy for cancer can cause significant gut toxicity leading to a breakdown of the selectivity of the mucosal barrier. This results in bacteria passing into the systemic circulation and causing serious symptomology such as oral mucositis, increased infection rates, and localized gut toxicity (diarrhea) [1,2]. Consequently, chemotherapy-induced gut toxicity (CIGT) is a significant burden on patients' quality of life and causes substantial additional health costs [3]. Research has focused on the mechanisms by which this toxicity occurs and has highlighted key roles for apoptosis [4], the microbiome and matrix metalloproteinases [2]. More recently an appreciation has been gained regarding the profound impact that the innate immune system has in creating CIGT, specifically via Toll-like receptors (TLRs) [5]. The gut is the largest immunological organ in the body, capable of generating immense immunological responses through activation of resident mucosal associated lymphoid tissue [6]. In the late 1990s, the pattern recognition receptor Toll was discovered, $[7,8]$ which led to the further characterization of the TLRs. Normal gut bacteria are tightly regulated via TLR signaling [9, 10]. TLRs are expressed within the epithelial cells of the small 
and large intestine [11], with research implicating TLRs as playing key roles in the maintenance of gut epithelial homeostasis [12]. Our previous research has demonstrated that upregulation of nuclear factor kappa $\mathrm{B}(\mathrm{NF}-\mathrm{kB})[13]$ and proinflammatory cytokines, [14] and alterations in epithelial cell proliferation [15] occur in the gut following chemotherapy. This implicates the role of TLR pathways being pivotal in the pathogenesis of CIGT and may offer new therapeutic targets to investigate. Further, TLR4 recognizes exogenous factors from invading microbes, endogenous danger signals, and some chemical structures. Thus, TLR4 may be uniquely positioned to be able to detect both the tissue damage generated as a consequence of chemotherapy, and the invading pathogens resulting from gut toxicity.

Aside from the substantial gut toxicity following chemotherapy, pain induced by chemotherapy is another adverse effect commonly experienced in the clinical setting [16, 17]. It can be one of the most important dose limiting complications, particularly in patients receiving taxane-, vinca alkaloid-, and/or platin-based chemotherapy [16, 17]. It is characteristically reported as a tingling, burning pain often accompanied with significant sensory-motor impairments $[18,19]$, is refractory to treatment in a large number of patients, and can persist as a chronic condition significantly impacting on the patients' quality of life [20].

The underlying mechanisms of the chemotherapy-induced pain are ill defined. It has been proposed that some cytotoxic agents may damage neurons through binding to axonal microtubules to subsequently alter axonal transport as shown with vincristine [21, 22]. However, chemotherapeutic agents are generally unable to cross the blood brain barrier and therefore should theoretically be unable to damage central neurons, strongly implying chemotherapy induces pain via other mechanisms. In addition to the direct damage to neurons as a result of chemotherapy exposure, the damage itself generates a "damaged tissue" signal, which would in turn result in localized glial activation and the subsequent secretion of proinflammatory cytokines to further exacerbate neuronal responses to potentiate pain [23].

The enteric nervous system is comprised of neurons and glial cells [24] and it is located within the gastrointestinal tract. Traditionally it has been thought enteric glia, which similarly to astrocytes in the central nervous system express increasing amounts of glial fibrillary acidic protein (GFAP) when activated, provide critical support and nutrition to the enteric neurons [24]. However, this has recently been expanded with research suggesting enteric glia regulate the intestinal epithelial barrier [25-27].

Our research [14, 28] and that of others [29] has strongly implicated the transcription factor NF- $\mathrm{KB}$ and proinflammatory cytokines including tumor necrosis factor (TNF- $\alpha$ ), interleukin (IL)-1 beta (IL-1 $\beta$ ), and IL-6, as central regulators of CIGT. Although it has been reported that taxol, which causes peripheral pain, also induced changes in the synthesis of pro-inflammatory cytokines including TNF- $\alpha$ and IL- $1 \beta$ [17], it remains unknown whether these same factors are key players in pain induced by other cytotoxics. Despite this common mechanism of these chemotherapyinduced toxic effects, to date, there has been no established link between the timing of pain and gut toxicity. Therefore, the aim of this study was to determine if there is a link between pain assessed by facial pain scoring [30], and gut toxicity assessed by diarrhea and weight loss. Our secondary aims were to determine if altered expression of TLRs, and specifically TLR4, is a key driver of these effects; and also to determine whether enteric nervous system GFAP expression is altered in response to chemotherapy.

\section{Materials and methods}

\section{Animals and ethics}

Female Dark Agouti (DA) rats, weighing between 150 and $170 \mathrm{~g}$ were used for this study. Rats were housed in Perspex cages at a temperature of $22 \pm 1{ }^{\circ} \mathrm{C}$ and subject to a $14 \mathrm{~h}$ light/ $10 \mathrm{~h}$ dark cycle. Animals had ad libitum access to autoclaved chow and water. Experimental design was approved by the Animal Ethics Committees of the Institute of Medical and Veterinary Science (IMVS), and The University of Adelaide, and complied with the National Health and Medical Research Council (Australia) Code of Practice for Animal Care in Research and Teaching.

\section{Experimental design}

Forty rats were randomly assigned to receive either irinotecan ( $n=5-8$ per time point, total of 34 ) or vehicle $(n=5)$. All rats received breast cancer inoculum as described previously [15]. In brief, rats were implanted with $4 \times 10^{6}$ cells in $0.2 \mathrm{~mL}$ phosphate-buffered saline (PBS) s.c. into each flank and tumors were allowed to grow for 1 week prior to administration of chemotherapy. All rats received $0.01 \mathrm{mg} / \mathrm{kg}$ s.c. atropine (to reduce the cholinergic reaction) immediately prior to administration of either $175 \mathrm{mg} / \mathrm{kg}$ i.p. irinotecan (kindly supplied by Pfizer, administered in a sorbitol/lactic acid buffer: $45 \mathrm{mg} /$ $\mathrm{mL}$ sorbitol $/ 0.9 \mathrm{mg} / \mathrm{mL}$ lactic acid, $\mathrm{pH} 3.4$ ), or vehicle (sorbitol/lactic acid buffer: $45 \mathrm{mg} / \mathrm{mL}$ sorbitol $/ 0.9 \mathrm{mg} / \mathrm{mL}$ lactic acid, $\mathrm{pH} 3.4$, previously shown to have no gut toxicity effects [31]) at time $0 \mathrm{~h}$. Groups of rats were killed using $3 \%$ isofluorane in $100 \% \mathrm{O}_{2}$ anesthesia and cervical dislocation at times $6,24,48,72,96$, and $120 \mathrm{~h}$ post-irinotecan treatment. The entire gastrointestinal tract (from the pyloric sphincter to the rectum) was dissected out and separated into the small intestine (pyloric sphincter to ileocecal sphincter) and colon (ascending colon to rectum). The small intestines and colons 
were flushed with chilled, sterile saline (Baxter Healthcare), and $1 \mathrm{~cm}$ samples dissected from $50 \%$ of the length of each, and fixed in $10 \%$ neutral-buffered formalin, processed and embedded in paraffin for immunohistological analyses.

\section{Gut toxicity assessment}

Gut toxicity was assessed through weight loss and occurrence of diarrhea as previously published as being associated with histological damage in the small and large intestine [32]. These measures were recorded on a daily basis including baseline. Animals were weighed daily at the same time, and total weight loss/gain recorded. Further, diarrhea occurrence and severity was recorded four times daily according to previous grading [31]: 0 , no diarrhea; 1 , mild diarrhea (staining of anus); 2 , moderate diarrhea (staining spreading over top of legs); and 3, severe diarrhea (staining over legs and abdomen, often with continual anal leakage). All gut toxicity assessments were conducted in a blinded fashion (RJG).

\section{Chemoneuropathy: facial pain assessment}

Chemoneuropathy was assessed four times daily using the established facial pain model that scored orbital tightening, nose bulge, cheek bulge, whisker change, and ear position [30]: 0 , not present; 1 , moderate; and 2, severe. All facial pain assessments were conducted in a blinded fashion (RJG).

\section{Immunohistochemistry}

\section{Toll-like receptors}

Sections of jejuna and colon were cut from paraffin blocks at $4 \mu \mathrm{m}$ thickness and mounted onto silane-coated slides (Lomb Scientific Pty Ltd). The jejuna was chosen as a representative region of the small intestine as in our model, irinotecan causes equivalent damage along the entire length of the small intestine as well as the large intestine [31,32]. Tissue samples were dewaxed in xylene (Merck Pty Ltd) and rehydrated through a graded series of ethanol (Chem Supply). Sections were immersed in $10 \mathrm{mM}$ citrate buffer (pH 6.0, Sigma-Aldrich Pty Ltd) and antigen retrieval performed by heating sections in a microwave (LG) on high $(800 \mathrm{~W})$ until boiling and then on low $(160 \mathrm{~W})$ for $10 \mathrm{~min}$. Sections were allowed to cool and endogenous peroxidise activity subsequently blocked with $3 \% \mathrm{H}_{2} \mathrm{O}_{2}$ in methanol (Chem Supply). Non-specific antibody binding was blocked with $20 \%$ normal goat or horse serum (NGS or NHS) as appropriate (Sigma-Aldrich Pty Ltd) in PBS for $30 \mathrm{~min}$ at room temperature (RT). Avidin-Biotin Blocking Kit (Vector Laboratories) was used to block endogenous avidin-biotin activity. Sections were incubated overnight with primary rat polyclonal antibodies (diluted in $5 \%$ NGS or NHS) directed at TLR2 at 1:50 (Novus Biologicals,
\#NB200-536), TLR4 at 1:50 (Abcam, \#ab13556), TLR5 1:50 (Abcam, \#ab62460) and TLR9 at 1:50 (Abcam, \#ab12121) at $4{ }^{\circ} \mathrm{C}$. Sections were then incubated with the appropriate secondary antibody (20 min at RT), ABC labeling reagent $(30 \mathrm{~min}$ at $\mathrm{RT})$ and developed with $3,3^{\prime}$ Diaminobenzidine. Sections were counterstained with LillieMayer's hematoxylin (HDS Scientific Supplies Pty Ltd), dehydrated and cleared in xylene (Merck Pty Ltd) before being mounted.

\section{GFAP}

Sections of jejuna and colon were cut from paraffin blocks at $4 \mu \mathrm{m}$ thickness and mounted onto silane-coated slides (Lomb Scientific Pty Ltd). Tissue samples were dewaxed in xylene (Merck Pty Ltd) and rehydrated through a graded series of ethanol (Chem Supply). Sections were immersed in $10 \mathrm{mM}$ citrate buffer (pH 6.0, Sigma-Aldrich Pty Ltd) and antigen retrieval performed by heating sections in a microwave (LG) on high $(800 \mathrm{~W})$ until boiling and then on low $(160 \mathrm{~W})$ for $10 \mathrm{~min}$. Sections were allowed to cool and endogenous peroxidise activity subsequently blocked with $3 \% \mathrm{H}_{2} \mathrm{O}_{2}$ in methanol (Chem Supply). Non-specific antibody binding was blocked with $20 \%$ normal horse serum (NHS) (SigmaAldrich Pty Ltd) in PBS for $30 \mathrm{~min}$ at room temperature (RT). Avidin-Biotin Blocking Kit (Vector Laboratories) was used to block endogenous avidin-biotin activity. Sections were incubated overnight with a primary goat polyclonal antibody (diluted in 10\% NHS) directed at GFAP at 1:400 (Santa Cruz Biotechnology, \#SC-6171) at $4{ }^{\circ} \mathrm{C}$. Sections were then incubated with an appropriate secondary antibody (20 min at RT), ABC labelling reagent (30 min at RT) and developed with 3,3'-Diaminobenzidine. Sections were counterstained with Lillie-Mayer's hematoxylin (HDS Scientific Supplies Pty Ltd), dehydrated and cleared in xylene (Merck Pty Ltd) before being mounted.

\section{Analysis}

Semi-quantitative immunohistochemistry was performed, with staining intensity graded as follows [33]: 0 , no staining; 1 , weak staining; 2 , moderate staining; 3 , strong staining; and 4 , intense staining. All assessments were done in a blinded fashion (RJG).

\section{Statistics}

All data for diarrhea, weight loss, facial pain, TLR2/4/5/9, and GFAP expression was compared to baseline. Comparisons of diarrhea severity scores (a single score in each $24 \mathrm{~h}$ period), percent weight loss, facial pain scores, TLR2/4/5/9, and GFAP expression over time was performed using the Kruskal-Wallis or one-way ANOVA tests as appropriate. Comparisons 
between facial pain scores in rats with and without diarrhea, and in rats with and without TLR expression were performed using the Mann-Whitney test. GraphPad Prism v 5.01 (GraphPad Software Inc, La Jolla, USA) was used for all statistical analysis. All data are presented as either median (range) or mean \pm SEM unless otherwise stated and $P<0.05$ was considered statistically significant.

\section{Results}

\section{Irinotecan caused significant gut injury and pain}

All rats receiving irinotecan developed gut injury evidenced by significant diarrhea and significant weight loss. Diarrhea occurred in a biphasic response, with symptoms appearing at $6 \mathrm{~h}$ firstly before resolving and then maximal symptoms observed at $72 \mathrm{~h}$ post-irinotecan (Fig. 1). Rats receiving vehicle control did not develop diarrhea at any time point.

Peak weight loss post-irinotecan compared to baseline occurred at $72 \mathrm{~h}($ mean $\pm \mathrm{SD}=11.1 \pm 6.6 \%, P<0.0001)$ before recovery at $120 \mathrm{~h}$ (mean $\pm \mathrm{SD}=-0.25 \pm 6.7 \%$ ) (Fig. 2). Rats receiving vehicle control continued to gain weight over the course of the experiment (Fig. 2).

Facial pain scores (Fig. 3), used as an indicator of pain, varied over time with peak pain occurring $72 \mathrm{~h}$ postirinotecan, median (range) $=5(0-5)$ versus $0(0-0)$ at baseline, $P<0.0001$. Recovery occurred by $120 \mathrm{~h}$ after irinotecan $(0(0-0))$. Rats receiving vehicle control only exhibited facial pain immediately after anesthetic and i.p. injections; these resolved after $6 \mathrm{~h}$ and did not reoccur over the course of the experiment. Importantly, there was also an association between the occurrence of facial pain and gut injury, such that rats with diarrhea had significantly higher facial pain scores compared to those without diarrhea: median (range) of 2.0 ( 0 to 5$)$ versus 0 ( 0 to 5) $(P=0.01)$.

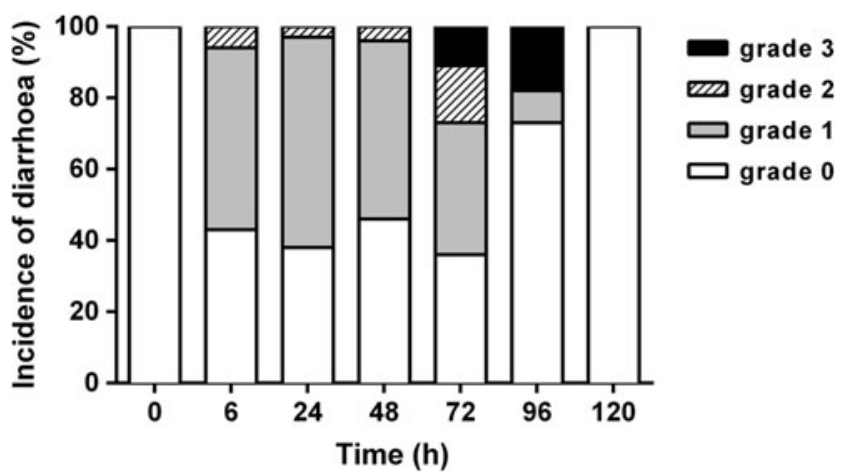

Fig. 1 Percentage of rats with grade mild, moderate, and severe diarrhea following irinotecan (175 mg/kg i.p.) administration

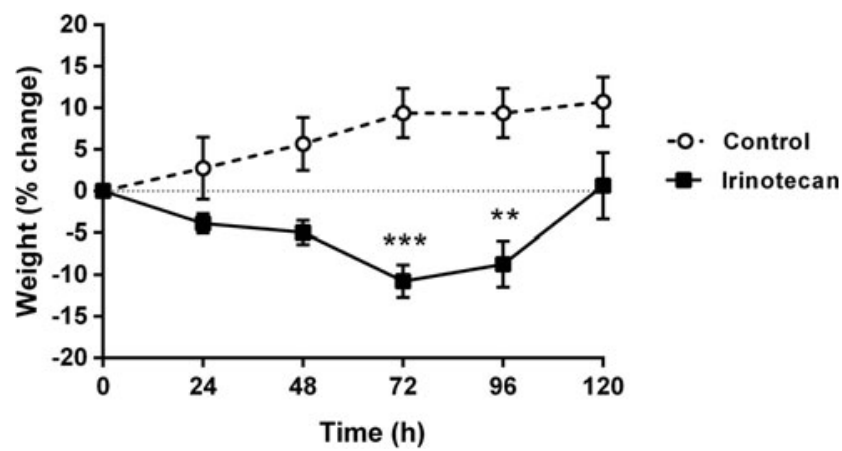

Fig. 2 Percentage change in weight from baseline to $120 \mathrm{~h}$ in rats following vehicle control or irinotecan $\left(175 \mathrm{mg} / \mathrm{kg}\right.$ i.p.). ${ }^{* *} P<0.01$ versus $120 \mathrm{~h},{ }^{*} * * P<0.0001$ versus $24 \mathrm{~h}$ and $120 \mathrm{~h}$

\section{Irinotecan decreased TLR4 and TLR5 expression} in the jejuna, with TLR4 expression associated with pain

There was no significant change in epithelial cell expression of TLR2 or TLR9 following irinotecan in either the jejuna or colon regions $(P=0.06-0.76)$. In contrast, both TLR4 and TLR5 expression was significantly decreased following irinotecan in the jejuna. More specifically, although TLR4 and TLR5 expression was unchanged across all time points in the epithelial cells of the villi of the jejuna, expression significantly decreased in jejunal crypts at 96 and $120 \mathrm{~h}$ after irinotecan $(P<0.001$, Fig. 4a TLR4, and $P<0.008$, Fig. $4 \mathrm{~b}$ TLR5). In contrast, TLR4 and 5 expression in the apical and basal colon remained unchanged across all time points $(P=$ 0.16-0.57). Staining was cytoplasmic throughout the study. Comparison of toxicity measures and TLR4 and TLR5 expression in the jejuna revealed that those rats with no TLR4 expression experienced less pain than those that expressed TLR4: median (range) $=0(0-5)$ versus $2(0-5), P=0.012$. No other significant associations were observed.

\section{Irinotecan increased GFAP expression in jejuna and colon}

GFAP was analyzed as a marker of altered enteric nervous system function. In the jejunum, GFAP expression was not detected in any control animal (Fig. 5). Further, there was no

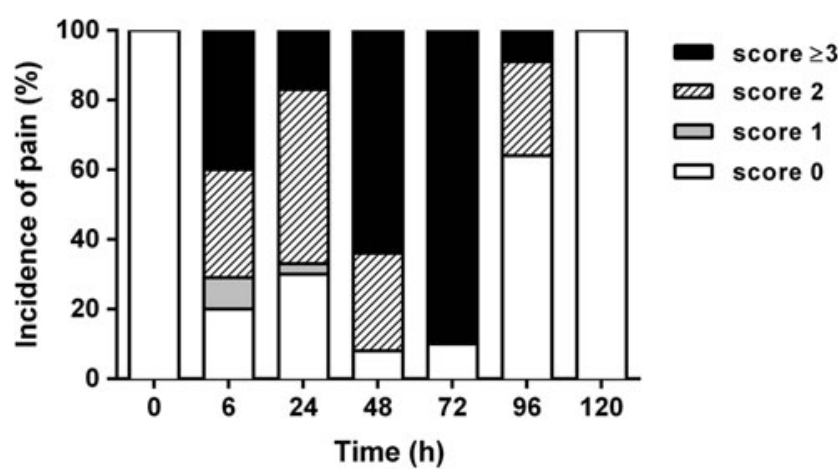

Fig. 3 Percentage of rats with facial pain scores of $0,1,2$, and $\geq 3$ between 6 and $120 \mathrm{~h}$ following irinotecan $(175 \mathrm{mg} / \mathrm{kg}$ i.p.) administration 
Fig. 4 Immunohistochemical staining intensities for TLR4 (a) and TLR5 (b) expression in jejuna of rats 6 to $120 \mathrm{~h}$ following irinotecan (175 mg/kg i.p.) administration compared to vehicle control. $* P<0.05$ versus control. Data are median (range). Representative images of TLR4 (c) and TLR5 (d)

immunohistochemical staining $(\times 200$ original magnification, scale bar length $=50 \mu \mathrm{m})$
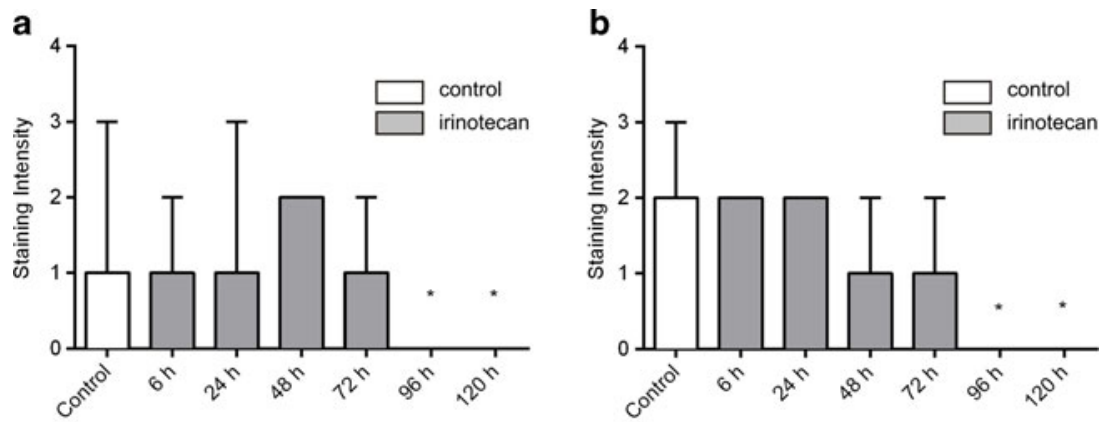

c
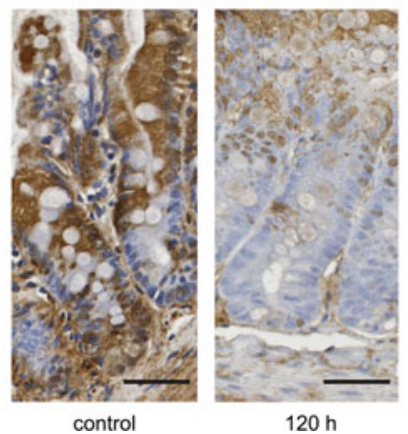

d



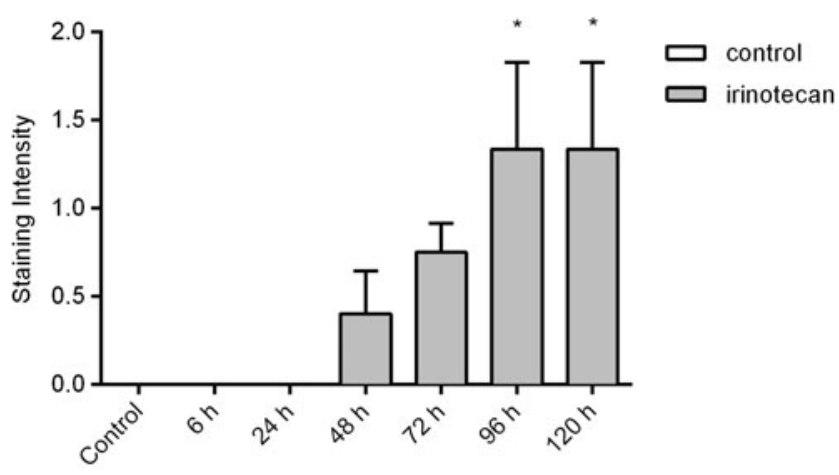

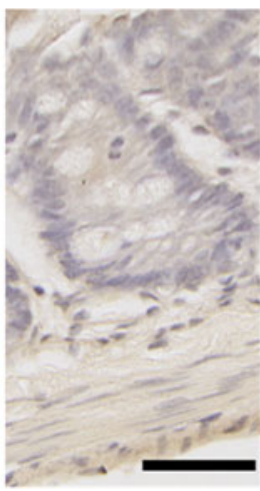

Control

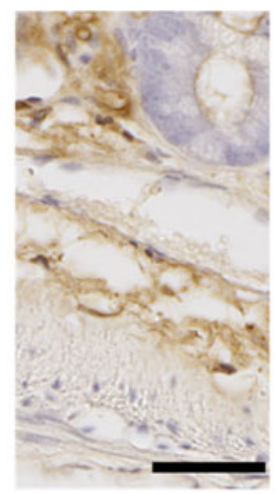

$120 \mathrm{~h}$
Fig. 5 Immunohistochemical staining intensities for GFAP expression in jejuna of rats 6 to $120 \mathrm{~h}$ following irinotecan $(175 \mathrm{mg} / \mathrm{kg}$ i.p.) administration compared to vehicle control. ${ }^{*} P<0.05$ versus control. Data are median (range). Representative images of immunohistochemical staining in vehicle control and $120 \mathrm{~h}$ irinotecan treated rats are also given $(\times 200$ original magnification, scale bar length $=50 \mu \mathrm{m}$ )
GFAP expression seen in the jejuna of animals killed 6 and $24 \mathrm{~h}$ after irinotecan administration. Mild GFAP expression was seen in animals killed $48 \mathrm{~h}$ and this continued to increase at $72 \mathrm{~h}$ peaking and remaining high at 96 and $120 \mathrm{~h}$ after irinotecan $(P=0.017)$. In contrast, in the colon, GFAP expression was detected in all animals, although expression was significantly higher 24 and $96 \mathrm{~h}$ after irinotecan expression $(P=0.04)$. GFAP staining was localized to the lamina propria and the submucosa.

\section{Discussion}

Both CIGT and pain are frequent, debilitating and doselimiting adverse effects of anti-cancer cytotoxic therapies $[17,20]$ affecting a large number of cancer patients. The present study has demonstrated that both toxicities have a similar time course of presentation, with peak gut toxicity and pain occurring at $72 \mathrm{~h}$ following chemotherapy. This implies that the underlying pathobiology of the two conditions may be linked in this preclinical model. In further support of this, we have also demonstrated that GFAP expression within the jejuna increases following chemotherapy, peaking at the later time points and remaining high during the healing phase of chemotherapy-induced gut toxicity. This increase in GFAP expression, indicative of glial activation, is in accordance with previous research that suggests enteric glia enhance epithelial restitution [26].

The currently accepted pathogenesis of CIGT is an intertwined five-phase model, characterized by dynamic 
biochemical interactions between chemotherapeutic agent(s) and the cellular constituents of the mucosa [34]. More specifically, NF- $\mathrm{KB}$ is a key transcription factor that plays a critical role in the pathogenesis. Our previous studies in this DA rat model of chemotherapy-induced gut toxicity have clearly shown NF-kB to be up-regulated by over twofold in the gut mucosa [13]. Further, as NF-kB modulates gene expression of pro-inflammatory cytokines, including TNF, IL-6, and IL- $1 \beta$, it is not surprising that we have also observed elevated levels of TNF, IL- $1 \beta$, and IL- 6 in the serum and tissue with our model [35].

Previous research has shown the enteric nervous system plays a key role in the maintenance of intestinal barrier homeostasis [36]. Enteric glial cells of the gut are specifically activated by pro-inflammatory cytokines, specifically IL-1 $\beta$, and IL-6 [24], which are known to be elevated early after the administration of chemotherapy, and are key players in development of chemotherapy-induced gut toxicity [13]. Enteric glial cells are also thought to produce IL- $1 \beta$ however the underlying mechanisms remain unknown [24]. Thus, we suggest pro-inflammatory cytokines, elevated early after chemotherapy and involved in the development of CIGT, also activate enteric glia, represented here by an increase in GFAP expression, which then play a role in restoring the damaged epithelium.

Chemotherapy-induced pain is one of the key dose limiting factors for many chemotherapy patients [17], leading to a reduced chance of cure. To date, there is no clear mechanism through which cytotoxic agents induce pain [17], and importantly hyperalgesia. However, chemotherapeutic agents have been demonstrated to cause the upregulation of IL-1 $\beta$ [37], a crucial mediator of neuropathic pain [38-40]. Thus, it is tangible to suggest that chemotherapy-induced pain may have the same key immune mediators underlying its pathogenesis as CIGT. However, to date, the link joining these two toxicities has been missing.

The observations from this current study indicate that this link may be involvement of TLRs, as evidenced by relationships between gut toxicity and pain, and TLR4 expression and pain. These data are similar to that reported in previous studies. For example, rats receiving cisplatin had a significant weight loss accompanied by changes in pain from mechanical and thermal tests [16]. TLR3, TLR4, and myeloid differentiation primary response 88 (MyD88, signaling mediator for TLR4) knockout mice had lower levels of cisplatin-induced pain from mechanical tests compared to wild-type mice, and there was a total absence of pain in double knockout mice (TIR-domain-containing adapter-inducing interferon- $\beta$ (Trif, signaling mediator for TLR4/MyD88)) [41]. While taxol treated macrophages from TLR4 and MyD88 knockout mice secreted minor amounts of pro-inflammatory mediators compared to macrophages from wild-type mice [42]. TLR4 signaling is known to activate NF- $\mathrm{BB}$, and therefore could be the key initiating factor in the underlying pathogeneses of both chemotherapy-induced gut toxicity and pain. Although in the present study TLR4 expression did not change during peak gut damage, it was completely absent during recovery phase in agreement with the inverse relationship between TLR/ $\mathrm{NF}-\mathrm{KB}$ and the $\mathrm{Wnt} / \beta$-catenin signal transduction pathway. It is known that $\mathrm{Wnt} / \beta$-catenin is "switched off" during times of pro-inflammation [43], but activated during periods of intestinal growth [44], such as during intestinal recovery following chemotherapy which we have shown to be a time of rapid cell division [31], hence explaining TLR4 absence during the healing phase. Further, as TLR $4 / 5$ expression was absent from the jejuna during recovery from gut toxicity, pharmacological inhibition of TLRs may be of clinical use to increase healing in the jejuna following chemotherapy.

In conclusion, this is the first study to show a close link between chemotherapy-induced pain and diarrhea, indicating a possible common underlying trigger and mechanism; here we provide evidence for altered TLR signaling pathways and increases in enteric nervous system GFAP expression. Furthermore, we have shown that TLR4 and TLR5 are specifically downregulated during gastrointestinal tissue recovery and pain resolution, whereas GFAP expression is significantly upregulated during gastrointestinal recovery. This suggests that feedback on these receptors occurs to facilitate healing and tissue restoration. Further research is now required to investigate the impact of TLR modulation in the development and resolution of chemotherapy-induced toxicity.

Acknowledgments The authors would like to thank Nadia Gagliardi and Chris Leigh from the School of Medical Sciences for technical assistance with immunohistochemistry staining and Masooma Sultani, Emma Bateman, and Andrea Stringer for assistance with the animal study.

Statement of author contributions All authors participated in the design, interpretation of the studies, analysis of the data, and manuscript review; RJG, JKC, HRW, and JMB conducted the experiments.

\section{Compliance with ethical standards}

Financial disclosure Associate Professor Rachel Gibson provides consultancy services and receives contract research funding from Onyx Pharmaceuticals. Dr. Joanne Bowen receives contract research funding from Pfizer and Puma Biotechnology.

Conflict of interests The authors declare that they have no competing interests.

Funding acknowledgement This work was supported by Cure Cancer/Cancer Australia [APP553601] and the Department for Science and Technology, South Australian Government [South Australian Professional Development Scholarship] awarded to Associate Professor Rachel Gibson. Dr. Janet Coller was the recipient of an FTT Fricker Fellowship (University of Adelaide, Medical Endowment Funds); Ms. Hannah Wardill is the recipient of the Australian Postgraduate Award and the Florey Medical Research Foundation Doctor Chun Chung and Madam So Sau Lam Memorial Top Up Scholarship for Cancer Research; Professor Mark Hutchinson is the recipient of an ARC Fellowship 
[DP110100297]; and Dr. Joanne Bowen was the recipient of an NHMRC Post-Doctoral Training Fellowship [539805].

Ethical approval All applicable international, national, and/or institutional guidelines for the care and use of animals were followed. All procedures performed in studies involving animals were in accordance with the ethical standards of the institution or practice at which the studies were conducted.

Experimental design was approved by the Animal Ethics Committees of the Institute of Medical and Veterinary Science (IMVS), and The University of Adelaide, and complied with the National Health and Medical Research Council (Australia) Code of Practice for Animal Care in Research and Teaching.

\section{References}

1. Sonis ST (2007) Pathobiology of oral mucositis: novel insights and opportunities. J Support Oncol 5(9 Suppl 4):3-11

2. Stringer AM, Al-Dasooqi N, Bowen JM, Tan TH, Radazum M, Logan RM, Mayo B, Keefe DMK, Gibson RJ (2013) Potential biomarkers of chemotherapy-induced diarrhea: a clinical study of intestinal microbiome alterations; intestinal inflammation and circulating matrix metalloproteinases. Support Care Cancer 21:18431852

3. Carlotto A, Hogsett VL, Mairorini EM, Razulis JG, Sonis ST (2013) The economic burden of toxicities associated with cancer treatment: review of the literature and analysis of nausea and vomiting, diarrhoea, oral mucositis and fatigue. Pharmacoeconomics 31:753-766

4. Keefe DM, Brealey J, Goland GJ, Cummins AG (2000) Chemotherapy for cancer causes apoptosis that precedes hypoplasia in crypts of the small intestine in humans. Gut 47:632-637

5. Wardill HR, Van Sebille YZA, Mander KA, Gibson RJ, Logan RM, Bowen JM, Sonis ST (2015) Toll-like receptor 4 signaling: a common biological mechanism of regimen-related toxicities: an emerging hypothesis for neuropathy and gastrointestinal toxicity. Cancer Treat Rev 41:122-128

6. Kelly D, Campbell JI, King TP, Grant G, Jansson EA, Coutts AG, Pettersson S, Conway S (2004) Commensal anaerobic gut bacteria attenuate inflammation by regulating nuclear-cytoplasmic shuttling of PPAR-gamma and RelA. Nat Immunol 5:104-112. doi:10.1038/ ni1018

7. Medzhitov R, Preston-Hurlburt P, Janeway CA Jr (1997) A human homologue of the Drosophila Toll protein signals activation of adaptive immunity. Nature 388:394-397. doi:10.1038/41131

8. Lemaitre B, Nicolas E, Michaut L, Reichhart JM, Hoffmann JA (1996) The dorsoventral regulatory gene cassette spatzle/Toll/cactus controls the potent antifungal response in Drosophila adults. Cell 86:973-983

9. Lee J, Mo JH, Katakura K, Alkalay I, Rucker AN, Liu YT, Lee HK, Shen C, Cojocaru G, Shenouda S, Kagnoff M, Eckmann L, BenNeriah Y, Raz E (2006) Maintenance of colonic homeostasis by distinctive apical TLR9 signalling in intestinal epithelial cells. Nat Cell Biol 8:1327-1336. doi:10.1038/ncb1500

10. Umesaki Y, Setoyama H (2000) Structure of the intestinal flora responsible for development of the gut immune system in a rodent model. Microbes Infect 2:1343-1351

11. Abreu MT (2010) Toll-like receptor signalling in the intestinal epithelium: how bacterial recognition shapes intestinal function. Nat Rev Immunol 10:131-144
12. Rakoff-Nahoum S, Paglino J, Eslami-Varzaneh F, Edberg S, Medzhitov R (2004) Recognition of commensal microflora by toll-like receptors is required for intestinal homeostasis. Cell 118: 229-241

13. Logan RM, Stringer AM, Bowen JM, Gibson RJ, Sonis ST, Keefe DM (2008) Serum levels of NFkappaB and pro-inflammatory cytokines following administration of mucotoxic drugs. Cancer Biol Ther 7:1139-1145

14. Logan RM, Gibson RJ, Bowen JM, Stringer AM, Sonis ST, Keefe DM (2008) Characterisation of mucosal changes in the alimentary tract following administration of irinotecan: implications for the pathobiology of mucositis. Cancer Chemother Pharmacol 62:3341. doi:10.1007/s00280-007-0570-0

15. Gibson RJ, Keefe DM, Thompson FM, Clarke JM, Goland GJ, Cummins AG (2002) Effect of interleukin-11 on ameliorating intestinal damage after methotrexate treatment of breast cancer in rats. Dig Dis Sci 47:2751-2757

16. Cata JP, Weng HR, Dougherty PM (2008) Behavioral and electrophysiological studies in rats with cisplatin-induced chemoneuropathy. Brain Res 1230:91-98. doi:10.1016/j.brainres.2008.07.022

17. Cata JP, Weng HR, Dougherty PM (2008) The effects of thalidomide and minocycline on taxol-induced hyperalgesia in rats. Brain Res 1229:100-110. doi:10.1016/j.brainres.2008.07.001

18. Boogerd W, ten Bokkel Huinink WW, Dalesio O, Hoppenbrouwers WJ, van der Sande JJ (1990) Cisplatin induced neuropathy: central, peripheral and autonomic nerve involvement. J Neurooncol 9:255263

19. Dougherty PM, Cata JP, Cordella JV, Burton A, Weng HR (2004) Taxol-induced sensory disturbance is characterized by preferential impairment of myelinated fiber function in cancer patients. Pain 109:132-142. doi:10.1016/j.pain.2004.01.021

20. Connelly E, Markman M, Kennedy A, Webster K, Kulp B, Peterson G, Belinson J (1996) Paclitaxel delivered as a 3-hr infusion with cisplatin in patients with gynecologic cancers: unexpected incidence of neurotoxicity. Gynecol Oncol 62:166-168. doi:10. 1006/gyno.1996.0210

21. Tanner KD, Levine JD, Topp KS (1998) Microtubule disorientation and axonal swelling in unmyelinated sensory axons during vincristine-induced painful neuropathy in rat. J Comp Neurol 395: 481-492. doi:10.1002/(SICI)1096-9861(19980615) 395:4<481::AID-CNE5>3.0.CO;2-Y

22. Weng HR, Cordella JV, Dougherty PM (2003) Changes in sensory processing in the spinal dorsal horn accompany vincristine-induced hyperalgesia and allodynia. Pain 103:131-138

23. Watkins LR, Maier SF, Goehler LE (1995) Immune activation: the role of pro-inflammatory cytokines in inflammation, illness responses and pathological pain states. Pain 63:289-302

24. Ruhl A, Nasser Y, Sharkey KA (2004) Enteric glia. Neurogastroenterol Motil 16(Suppl 1):44-49. doi:10.1111/j.17433150.2004.00474.x

25. Toumi F, Neunlist M, Cassagnau E, Parois S, Laboisse CL, Galmiche JP, Jarry A (2003) Human submucosal neurones regulate intestinal epithelial cell proliferation: evidence from a novel coculture model. Neurogastroenterol Motil 15:239-242

26. Van Landeghem L, Chevalier J, Mahe MM, Wedel T, Urvil P, Derkinderen P, Savidge T, Neunlist M (2011) Enteric glia promote intestinal mucosal healing via activation of focal adhesion kinase and release of proEGF. Am J Physiol Gastrointest Liver Physiol 300:G976-G987. doi:10.1152/ajpgi.00427.2010

27. Watson AJ, Duckworth CA, Guan Y, Montrose MH (2009) Mechanisms of epithelial cell shedding in the mammalian intestine and maintenance of barrier function. Ann N Y Acad Sci 1165:135142. doi:10.1111/j.1749-6632.2009.04027.x

28. Logan RM, Gibson RJ, Sonis ST, Keefe DM (2007) Nuclear factorkappaB (NF-kappaB) and cyclooxygenase-2 (COX-2) expression 
in the oral mucosa following cancer chemotherapy. Oral Oncol 43: 395-401. doi:10.1016/j.oraloncology.2006.04.011

29. Sonis ST (2002) The biologic role for nuclear factor-kappaB in disease and its potential involvement in mucosal injury associated with anti-neoplastic therapy. Crit Rev Oral Biol Med 13:380-389

30. Langford DJ, Bailey AL, Chanda ML, Clarke SE, Drummond TE, Echols S, Glick S, Ingrao J, Klassen-Ross T, Lacroix-Fralish ML, Matsumiya L, Sorge RE, Sotocinal SG, Tabaka JM, Wong D, van den Maagdenberg AM, Ferrari MD, Craig KD, Mogil JS (2010) Coding of facial expressions of pain in the laboratory mouse. Nat Methods 7:447-449. doi:10.1038/nmeth. 1455

31. Gibson RJ, Bowen JM, Inglis MR, Cummins AG, Keefe DM (2003) Irinotecan causes severe small intestinal damage, as well as colonic damage, in the rat with implanted breast cancer. $\mathrm{J}$ Gastroenterol Hepatol 18:1095-1100

32. Wardill HR, Bowen JM, Sultani M, Stringer AM, Stansborough R, Shirren J, Gibson RJ (2014) Irinotecan disrupts tight junction proteins within the gut: implications for chemotherapy-induced gut toxicity. Cancer Biol Ther 15:1-9

33. Al-Dasooqi N, Gibson RJ, Bowen JM, Logan RM, Stringer AM, Keefe DM (2010) Matrix metalloproteinases are possible mediators for the development of alimentary tract mucositis in the Dark Agouti rat. Exp Biol Med (Maywood) 235:1244-1256. doi:10. 1258/ebm.2010.010082

34. Sonis ST (2004) A biological approach to mucositis. J Support Oncol 2:21-32, discussion 35-6

35. Logan RM, Stringer AM, Bowen JM, Gibson RJ, Sonis ST, Keefe DM (2009) Is the pathobiology of chemotherapy-induced alimentary tract mucositis influenced by the type of mucotoxic drug administered? Cancer Chemother Pharmacol 63:239-251. doi:10. 1007/s00280-008-0732-8

36. Neunlist M, Aubert P, Bonnaud S, Van Landeghem L, Coron E, Wedel T, Naveilhan P, Ruhl A, Lardeux B, Savidge T, Paris F,
Galmiche JP (2007) Enteric glia inhibit intestinal epithelial cell proliferation partly through a TGF-beta1-dependent pathway. Am J Physiol Gastrointest Liver Physiol 292:G231-G241. doi:10.1152/ ajpgi.00276.2005

37. Zaks-Zilberman M, Zaks TZ, Vogel SN (2001) Induction of proinflammatory and chemokine genes by lipopolysaccharide and paclitaxel (Taxol) in murine and human breast cancer cell lines. Cytokine 15:156-165. doi:10.1006/cyto.2001.0935

38. DeLeo JA, Yezierski RP (2001) The role of neuroinflammation and neuroimmune activation in persistent pain. Pain 90:1-6

39. Watkins LR, Maier SF (2003) Glia: a novel drug discovery target for clinical pain. Nat Rev Drug Discov 2:973-985

40. Ledeboer A, Liu T, Shumilla JA, Mahoney JH, Vijay S, Gross MI, Vargas JA, Sultzbaugh L, Claypool MD, Sanftner LM, Watkins LR, Johnson KW (2006) The glial modulatory drug AV411 attenuates mechanical allodynia in rat models of neuropathic pain. Neuron Glia Biol 2:279-291. doi:10.1017/S1740925X0700035X

41. Park HJ, Stokes JA, Corr M, Yaksh TL (2014) Toll-like receptor signaling regulates cisplatin-induced mechanical allodynia in mice. Cancer Chemother Pharmacol 73:25-34

42. Byrd-Leifer CA, Block EF, Takeda K, Akira S, Ding A (2001) The role of MyD88 and TLR4 in the LPS-mimetic activity of Taxol. Eur J Immunol 31:2448-2457. doi:10.1002/1521-4141(200108) 31:8<2448::AID-IMMU2448>3.0.CO;2-N

43. Neumann J, Schaale K, Farhat K, Endermann T, Ulmer AJ, Ehlers S, Relling N (2010) Frizzled1 is a marker of inflammatory macrophages, and its ligant Wnt3a is involved in reprogramming mycobacterium tuberculosis-infected macrophages. FASEB J 24:45994612

44. Camac KS, Thompson FM, Cummins AG (2007) Activation of beta-catenin in the stem cell region of crypts during growth of the small intestine in infant rats. Dig Dis Sci 52:1242-1246. doi:10. 1007/s10620-006-9200-7 